\title{
Future-proofing pathology: the case for clinical adoption of digital pathology
}

\author{
Bethany Jill Williams, ${ }^{1}$ David Bottoms, ${ }^{2}$ Darren Treanor ${ }^{3}$
}

${ }^{1}$ Department of Histopathology, St James' University Hospital, Leeds, UK

${ }^{2}$ North of England Clinical Networks, Richmond, UK ${ }^{3}$ Leeds Teaching Hospitals NHS Trust, University of Leeds, Leeds, UK

\section{Correspondence to}

Dr Bethany Jill Williams, Leeds Teaching Hospitals NHS Trust, University of Leeds, Department of Histopathology, Bexley Wing, St James' University Hospital, Beckett Street, Leeds, LS9 7TF, UK; bethany.williams2@nhs.net

Received 27 June 2017 Accepted 30 June 2017 Published Online First

5 August 2017

\section{CrossMark}

To cite: Williams $B J$,

Bottoms D, Treanor D.

J Clin Pathol

2017;70:1010-1018.

\section{ABSTRACT}

This document clarifies the strategic context of digital pathology adoption, defines the different use cases a healthcare provider may wish to consider as part of a digital adoption and summarises existing reasons for digital adoption and its potential benefits. The reader is provided with references to the relevant literature, and illustrative case studies. The authors hope this report will be of interest to healthcare providers, pathology managers, departmental heads, pathologists and biomedical scientists that are considering digital pathology, deployments or preparing business cases for digital pathology adoption in clinical settings. The information contained in this document can be shared and used in any documentation the reader wishes to present for their own institutional case for adoption report or business case.

\section{INTRODUCTION}

Digital pathology or whole slide imaging (WSI), the examination of digital slides on computer workstations, has long been recognised as an important healthcare education and research tool. More recently, healthcare providers have expressed increasing levels of interest in total or partial adoption of digital pathology in the diagnostic laboratory, accelerated by the recent Food and Drug Administration approval of a WSI device for primary diagnosis in the USA. ${ }^{1}$ To date, much data on real-world digital pathology deployment has come from diagnosticians in Europe and Canada, where a number of WSI devices have been marketed for primary diagnosis for a few years. Digital pathology has the potential to revolutionise the way in which pathology services are delivered, offering a flexible platform for safety, quality and efficiency improvements in diagnostic pathology, while future-proofing an increasingly pressured medical specialty.

This document aims to clarify the strategic context of digital pathology adoption, define the different use cases a healthcare provider may wish to consider as part of a digital adoption and summarise existing reasons for digital adoption and its potential benefits. The reader will be provided with references to the relevant literature, and illustrative case studies.

The document will be of interest to healthcare providers, pathology managers, departmental heads, pathologists and biomedical scientists that are considering digital pathology deployments, or preparing business cases for digital pathology adoption in clinical settings. The information contained in this document can be shared and used in any documentation the reader wishes to present for their own institutional case for adoption report or business case.

\section{STRATEGIC CONTEXT \\ Increasing volume and complexity of the histopathology workload}

Diagnostic pathology services face the challenge of an increasing workload, both in terms of volume and data complexity of cancer specimens and pressure to maintain or improve on diagnostic turnaround times. In the UK, year on year, the volume of cellular pathology requests received by laboratories has increased by an average of $4.5 \% .^{2}$

The drive to identify precancers and early stage cancers adds to the complexity of histopathological assessment, when morphology can be harder, and more time consuming to interpret. Pathologists are required to identify subtler pathologies in smaller diagnostic specimens. In addition to an increase in specimen requests, the pathologist is required to take more blocks and make more slides for each cancer specimen. These extra slides are required to satisfy the increasingly complex information requirements of the evidence-based cancer datasets of the Royal College of Pathology and the College of American Pathologists. ${ }^{2}$ In parallel with the increased complexity of standard morphological assessment required for cancer specimens, the battery of immunohistochemical and molecular tests which can aid with diagnosis, prognosis or therapeutic decision making is expanding year on year, again requiring more time from the pathologist.

\section{Pressure on turnaround times}

Challenging cancer turnaround targets are already in place in the National Health Service (NHS), with the 2015 report of the Independent Cancer Taskforce proposing even higher standards for turnaround times, such that by $2020,50 \%$ of patients referred for cancer testing by a GP will have their definitive diagnosis communicated to them within 2 weeks, and 95\% within 4 weeks. ${ }^{3}$ Increased demand has resulted in a negative impact on turnaround times, with data for England showing that the number of patients waiting more than 6 weeks for diagnostics in pathology has been increasing at approximately $17 \%$ per year since $2010-2011$, with the majority of delays waiting for histopathology. ${ }^{3}$

\section{Workforce crisis}

Against this background of escalating diagnostic workload, pathology is in the midst of an emergent 
workforce crisis. In the USA, it is predicted that the number of practicing pathologists will have declined from 5.7 to 3.7 per 100000 people between 2010 and $2030 .{ }^{4}$ In the next 5-10 years, there will be a similar shortage of consultant pathologists in the UK across all subspecialties. Data from the Royal College of Pathologists show that $32 \%$ of cellular pathologists are over the age of 55 (615 people), and are expected to retire in the next 5 years. $^{2}$

Meanwhile, from August 2015 to June 2016, only 52 trainees in histopathology were recommended to the General Medical Council for completion of training. Waiting times are starting to increase as a result of increasing mismatch between staffing capacity and demand.

\section{The need to increase capacity}

In the USA, it is predicted that pathologist workforce demand will have increased by $16 \%$ by $2030,{ }^{5}$ while numbers of pathologists per capita decline. In their November 2016 publication, 'Testing times ahead? An evaluation of capacity in pathology', Cancer Research UK highlights the need to ensure pathology services maximise efficiency, with networking and consolidation of pathology services prioritised. In light of increasing costs for staff overtime and outsourcing, optimisation of the pathology workforce is vital. Improved retention of near-retirement consultants, and increased efforts to drive recruitment in medical schools have been proposed, but these measures are not sufficient to solve the problem. The report recommends that departments and trusts should invest in infrastructure to support digital pathology, and that on-screen examination of histological slides should be used to enable more efficient networked services. This sentiment is echoed in the Nuffield Trust's publication- 'The Future of Pathology' which states that 'without change it will be difficult to maintain an adequately skilled workforce in many areas of the country'. ${ }^{6}$

\section{The drive towards networks and service mergers}

In the UK, the two Carter reviews of pathology, of 2006 and 2008 both recommended the formation of networked pathology services, with centralisation of laboratory resources, and the development of 'hub and spoke' local networks. ${ }^{78}$ More recently, the report by Lord Carter into operational productivity in the NHS suggested further consolidation and collaboration between services, stating that 'Our further analysis has confirmed that consolidated pathology organisations are the most efficient in the NHS'. ${ }^{9}$ Digital pathology offers an enabling platform for centralised slide production, with dispersal of diagnostic pathologists across or between regions. Digital imaging will assist trusts in the flexible use of clinical pathologist expertise in relation to laboratory locations, pathologist offices and MDT inputs. In their paper, 'Can Digital Pathology Result In Cost Savings? A Financial Projection for Digital Pathology Implementation at a Large Integrated Health care Organization', Ho et al describe how a digital pathology system would enable enterprise wide reporting of specimens, while allowing laboratory services to consolidate from 20 dispersed hospitals to 2 centralised sites. ${ }^{10}$

\section{The drive towards digitisation of healthcare}

As part of the Five-Year Forward View, the Independent Cancer Taskforce Strategy's paper, 'Achieving world class outcomes; a strategy for England 2015-20' highlights a drive towards achieving earlier diagnosis, the need to invest to deliver a modern, high-quality cancer service and the importance of training staff to realise advances in technology. ${ }^{311}$ The National
Information Board's Framework for Action 'Personalized Health and Care 2020', similarly emphasises the importance of improving access to healthcare records, implementing personalised medicine, supporting innovation and getting best use from technology. Digital pathology can take medicine a step further towards the information governance target of a 'paperless' healthcare system. ${ }^{12}$ The National Advisory Group on Health Information Technology's paper 'Making IT work-harnessing the power of health information technology to improve care in England' (August 2016) states the Five Year Forward View aims will not be met without prioritising digitisation of services, and that digitisation is likely to reap safety and quality improvements, concluding that 'the one thing the NHS cannot afford to do is to remain a largely non-digital system, it is time to get on with IT, ${ }^{13}$

\section{CLINICAL USES OF DIGITAL PATHOLOGY Individual use cases}

A number of potential use cases for digital pathology have been identified, and an individual healthcare provider can select which use cases are most relevant to their clinical needs and constraints.

\section{Primary diagnosis of pathological specimens}

This is the replacement of conventional light microscopic examination of glass slides with examination of whole slide images on screen by a pathologist to make a diagnosis as their standard diagnostic workflow. A diagnostic department may decide to use digital pathology for the primary diagnosis of the entirety of its workload, or may select individual histopathology subspecialties (eg, gynecopathology) or individual histopathologists' workloads to digitise. Kalmar and Linköping Hospitals in Sweden were early adopters of digital pathology, with slide scanning fully integrated into laboratory workflow, and primary diagnosis made on digital slides by a proportion of their pathologists. ${ }^{14}$

\section{Assessment of immunohistochemistry}

This is the replacement of conventional light microscopic examination of glass slides with examination of whole slide images by pathologists to assess immunohistochemical stains. Such immunohistochemical glass slides are often secondary/ancillary tests, which do not form part of the initial laboratory/diagnostic workflow for a case.

\section{MDT/tumour board}

The selection, collection, review and presentation of whole slide images or annotated regions of interest of cases for discussion at multidisciplinary meetings/tumour boards.

\section{Frozen section diagnosis}

The use of whole slide images to provide rapid, intraoperative histopathological opinion. The pathologist may be on site, or may be working remotely, particularly if the service is required out of hours. This use case for digital pathology has been successfully implemented in Canada for over 10 years, with neuropathology frozen sections reported remotely on digital slides since $2006 .{ }^{15} 16$

\section{Receiving second opinions/review cases}

The use of a digital pathology system to render a second opinion on a previously examined case, for example, a case submitted for MDT/tumour board discussion, a difficult skin case from a general pathologist to a dermatopathologist. 


\section{Requesting second opinions}

The use of a digital pathology system to request a second opinion on a previously examined case. Second opinions may be required: a) within a department for difficult cases (mandatory for reporting of certain entities, eg, dysplasia in patients with Barrett's oesophagus undergoing surveillance), ${ }^{17} \mathrm{~b}$ ) within a regional network for referral and review of MDT/tumour board cases, c) from regional/ national/international experts for rare/complicated cases and d) as part of quality assurance protocol within a department to audit and detect diagnostic errors.

\section{Remote working}

This is the use of a digital pathology system to enable diagnostic pathologists to view slides and make diagnoses from off-site locations, which may include other networked hospitals, academic institutions or home. Gävle hospital, Sweden, were able to solve a local recruitment problem by employing a pathologist to work remotely from home, receiving their workload in the form of digital slides. $^{18}$

\section{Insourcing/outsourcing diagnostic work}

The use of a digital pathology to allocate units of work across and beyond regional/national networks, or between public and private institutions to generate income, eliminate backlogs or make best use of available diagnosticians. On an international scale, digital pathology may help to broaden access to quality diagnostics, while offering income generating opportunities, a strategy already being explored at a number of centres, including the University of Pittsburgh Medical Center. ${ }^{19}$ Similarly, time-sensitive diagnostic slides could be outsourced to trusted partners in alternative time zones to enable rapid diagnostic turnaround out of hours, without resorting to costly and inconvenient pathologist on-call rotas.

\section{Deployment strategies}

Deployment strategies are likely to vary according to the strategic context, and local constraints of the institution, but a number of common scenarios for adoption can be described.

1. To solve a specific local staffing/logistical issue

A department may find it temporarily lacks sufficient diagnosticians in a particular field, either due to staff losses or increasing workload, for example, gynaecopathology. Introduction of a single scanner to scan all or part of the gynaecopathology workload might aid the department by (a) encouraging return or retention of periretirement diagnosticians, who will be able to work flexibly and remotely, (b) potentially attracting new workforce applicants that are interested in working digitally and (c) enabling rapid outsourcing of work to regional partners.

2. For a specific diagnostic purpose

For example, remote reporting of frozen section/urgent out of hours specimens. The ability to report these specimens remotely makes on call duties less onerous for the diagnostician, and could improve turnaround times of out of hours reporting, as consultants will not be required to travel to the hospital to make their diagnosis. The technology would also help in situations where the local specialist is not available to make a diagnosis (eg, due to illness, annual leave), and the slides can be transmitted to a regional partner.

3. For a fully digital diagnostic service

Digitisation of the diagnostic activities of a histopathology department may occur in a phased manner, allowing stepwise introduction of a number of use cases, and gradual accrual of experience with the technology. This would result in stepwise accumulation of the benefits of digitisation over a number of years. For an example of a phased deployment and the accumulation of benefits, see figure 1. Smaller, simpler changes in workflow with immediate efficiency savings, for example, second opinion cases, MDT digitisation

could be prioritised early in the digitisation, with more complex, large-scale changes in practice such as use of digital diagnosis for standard primary diagnosis deferred until the laboratory, and diagnosticians have gained experience in the laboratory workflow, and the digital diagnostic process.

4. For regional transformation

Digitisation may form part of larger, regional sustainability plan, with scanners and diagnostic workstations installed at a number of sites to form a regional network for collaboration, transmission of cases to MDT or for second opinion, redistribution of workload in response to fluctuations in demand and capacity. In this way, digitisation could underpin the structure of laboratory mergers and centralisation of laboratory services.

\section{BENEFITS OF DIGITAL PATHOLOGY ADOPTION-NON- FINANCIAL}

The principal perceived benefits of adoption of digital pathology can be broadly divided into four domains: improving patient safety, improving diagnostic workflow, improving workforce factors and improving service quality, with improvement in any one domain likely to contribute to improvement in all other domains (see figure 2). In this section, we will consider all the potential non-financial benefits of digital pathology, with references to evidence where appropriate.

\section{Improving patient safety}

Reduced risk of patient/slide misidentification errors

The use of an integrated digital pathology system, with paperless transmission of digital slides directly to the pathologist significantly reduces the possibility of a misidentification or transposition error (eg, mixing up slides from two patients). These are potentially the most serious errors that can originate in the diagnostic laboratory, with an incidence estimated at $1 \% .^{20}$ Digitisation of prescription practices, with the introduction of e-prescribing lead to a significant reduction in the relative risk of medication error of $13 \%-99 \% .^{21}$

\section{Reduced risk of tissue/slide loss or damage}

Potential loss and damage of valuable patient tissue on glass slides is a risk faced by laboratories on a daily basis as they transport glass from the laboratory to the diagnostician, from the feeder hospital to the regional cancer centre for review, or from the general pathologist to the recognised expert. Digital slides provide a portable, instantaneously transmissible diagnostic image which does not fade or degrade, and is not subject to the transport risks faced by glass slides.

\section{Improving the diagnostic workflow \\ Workload allocation}

A digital pathology system offers the flexibility and agility for streamlined 'pushing' and 'pulling' of cases to and by pathologists to respond to fluctuations in workload or case mix in a department. Digital slide management software can allow the entirety of a pathology workforce access to outstanding or backlogged work, enabling pathologists with extra capacity to 'pull' pooled cases. 


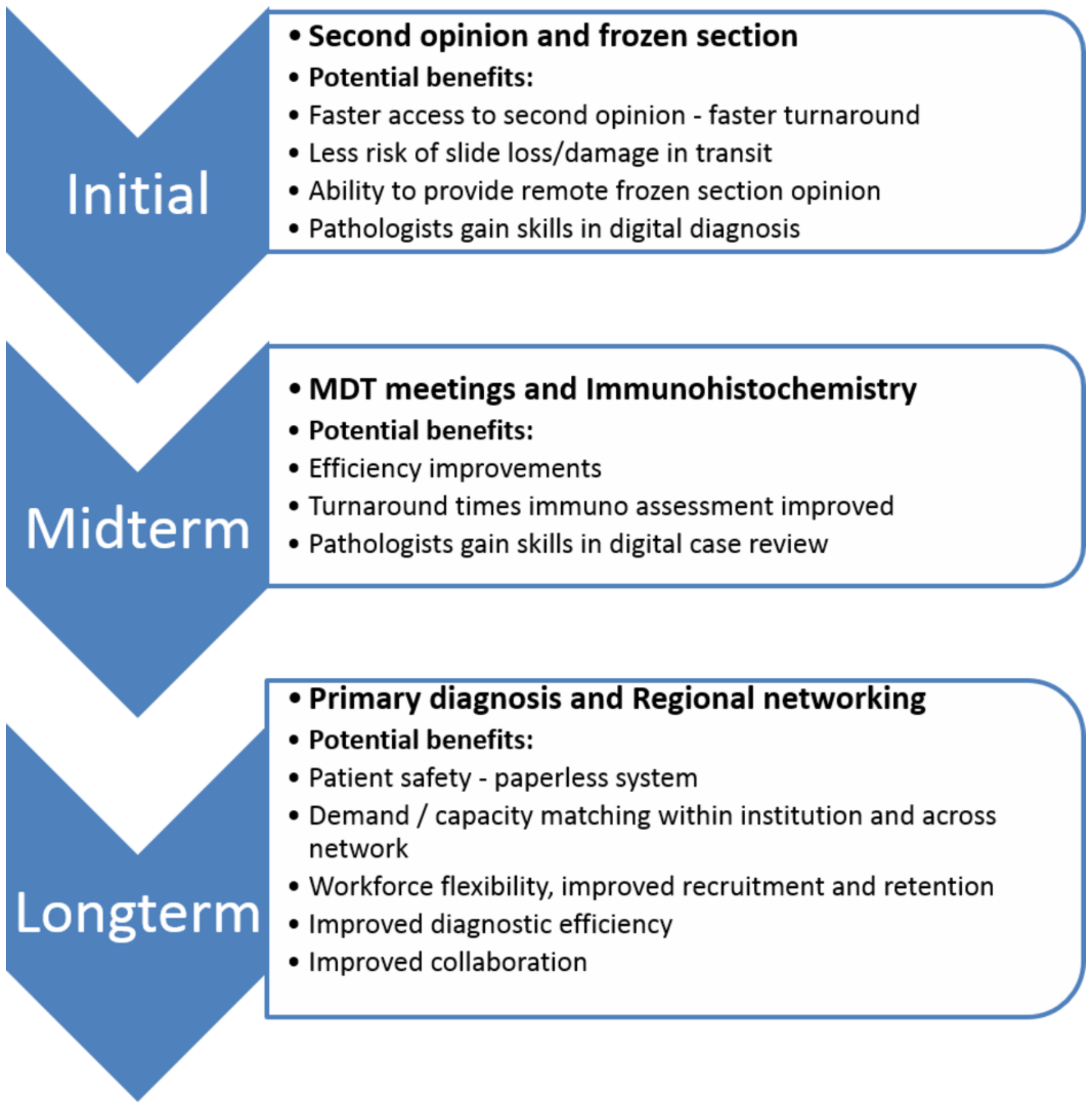

Figure 1 A phased approach to digitisation.

Conversely, a digital pathology system also allows for expedited 'pushing' of cases from a pool, or between pathologists, to ensure cases are promptly transferred to the most appropriate diagnostician within a network, or across a region.

Enabling flexible workload distribution, both within an institution and across a network allow for closer capacity-demand matching and a more lean approach to achieving the requisite diagnostic output for a population.

Rapid case tracking, archival and retrieval

In the conventional laboratory with glass slide diagnostics, trays of slides and request forms are delivered to and transferred between a variety of locations within the laboratory and the diagnostic department. There are ample opportunities for slides to get mislaid, and urgent sourcing of a glass slide can be time consuming for clerical and diagnostic staff. A digital system ensures that a crucial or time-sensitive case can be accessed instantly, by any registered user, should the need arise.

Review of previous specimens can be vital in cases such as the assessment of progressive disease or evaluation of a new tumour in a patient with cancer, and is likely to improve the quality of the pathologists' assessment of a live case. The storage of digital slides allows for instant retrieval and review of cases, a process which is time consuming and inefficient using conventional glass slide archives.

Increased diagnostic efficiency

One time and motion study identified potential for a $13 \%$ time saving in pathologist diagnostic efficiency with digital slide 
reporting, with efficiency gains in the organisation of, querying, matching and searching of cases. ${ }^{22}$ In addition, a limited number of diagnostic centres and individual diagnosticians have reported increased diagnostic efficiency using digital microscopes versus conventional light microscope. ${ }^{23}$ These improvements relate to a number of specific areas, including rapid availability of images, faster on-screen measurements and annotations of slides and ability to multitask while using a computer screen for diagnosis, instead of switching between the microscope and the PC. In addition, pathologists do not have to physically load and unload microscope slides, compare glass slide labels with paper request forms or refocus their microscopes for tissue of different thicknesses. While the existing literature shows promise, more work is needed in terms of large-scale clinical deployments before we have evidence of improved diagnostic efficiency.

Reduced case transfer times between the laboratory and the diagnostic pathologist

Current glass slide dependent processes rely on delivery or collection of assembled cases of glass slides from the laboratory, an inefficient process requiring time and manpower, which risks loss or damage of slides. With a digital pathology system, slides are instantaneously accessible to diagnosticians without the need for physical case assembly and delivery.

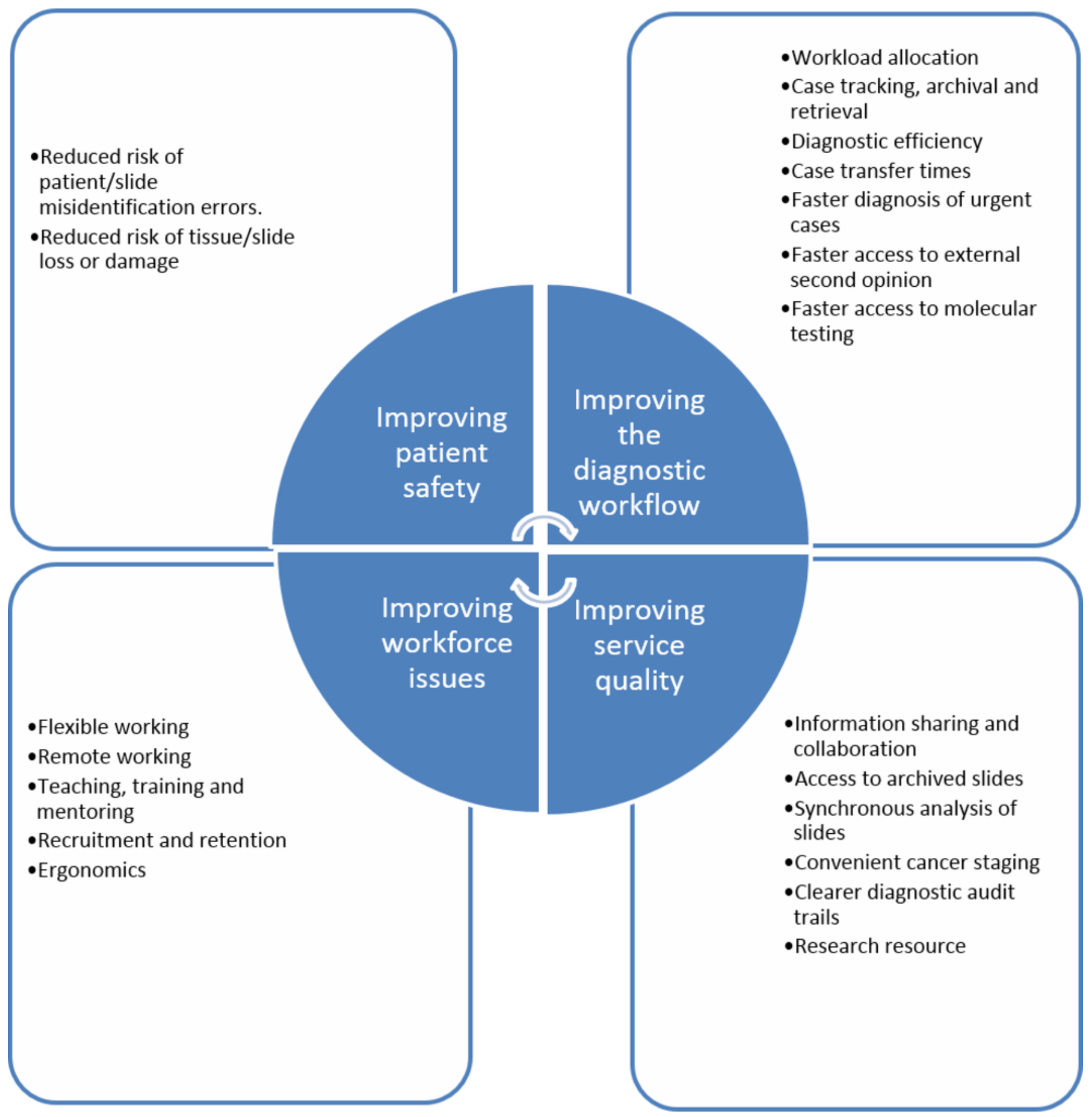

Figure 2 The benefits of digital pathology. 


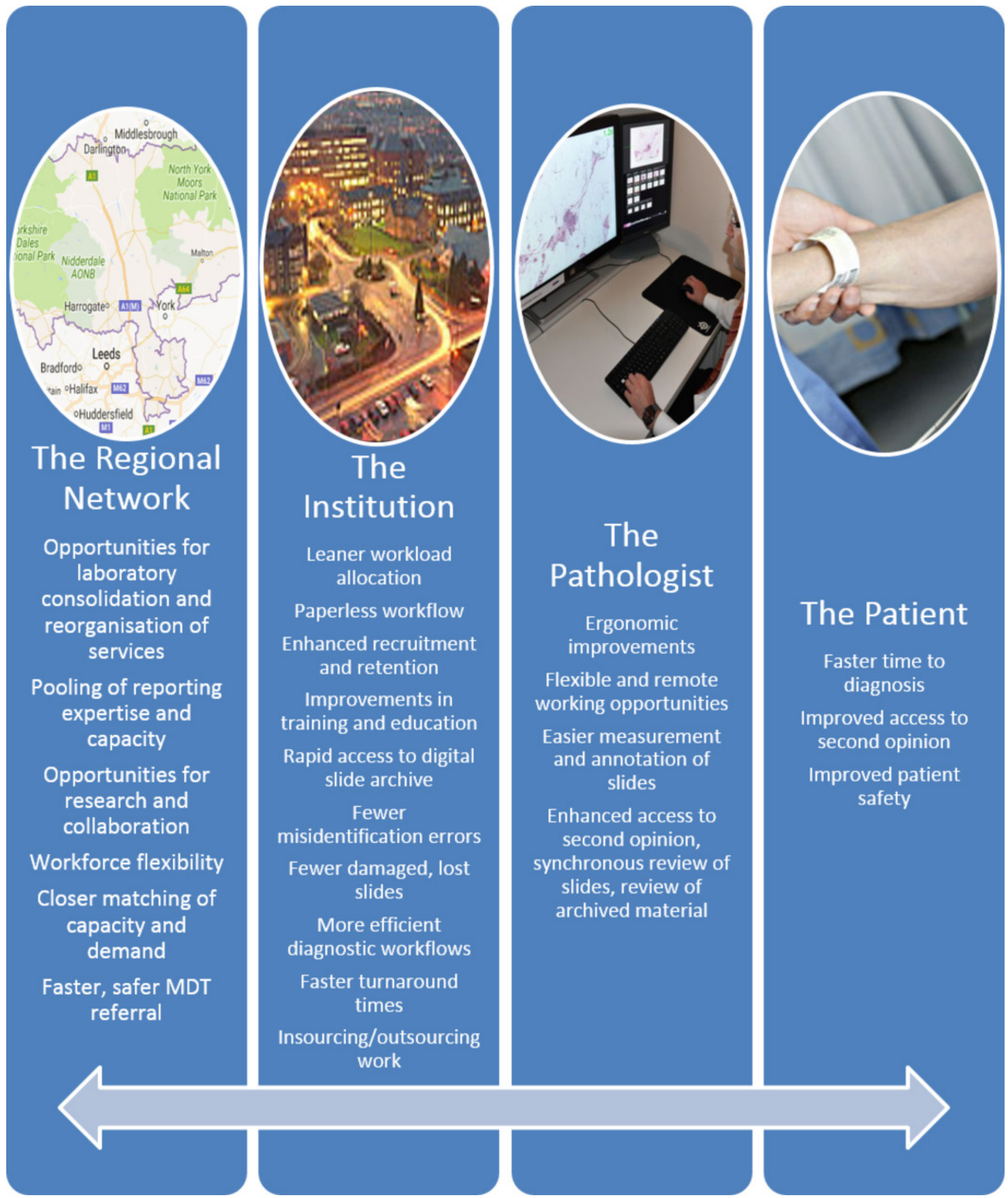

Figure 3 The benefits of digital pathology at multiple levels.

Faster diagnosis of urgent cases

Prioritisation of urgent cases can be difficult to manage using conventional glass slide processes, and is often reliant on manual tagging or labelling of specimens as urgent. This can be difficult to do when slides are in transit, in pools or on pathologists' desks. Recategorisation or escalation of case urgency is difficult and time consuming. Digital pathology allows easy flagging and escalation of priority of cases, and enables the laboratory administrator to 'push' the most urgent cases to the top of pathologist's worklists, without the need for explicit communication. 
Faster access to external second opinion

Substantial numbers of slides are transferred between hospitals, either as submissions for MDT discussion at regional cancer centres, or for second opinion of difficult or rare entities from recognised specialists. Faster turnaround times of cancer specimens are likely to lead to improved quality of cancer diagnosis and care. The Royal College of Pathologists, through Good Pathology Practice, ${ }^{24}$ recommends that all pathologists should actively participate in referral practice as this is in the best interests of patients, good continuing professional development and good practice, and that financial considerations should not be a deterrent to referral.

\section{Faster access to molecular/ancillary testing}

Digital pathology provides a platform for parallel specimen workflows between histopathology and molecular medicine. When the pathological assessment of a case is liberated from stained tissue on glass, the glass and tissue can be expedited to molecular medicine where appropriate, converting a sequential histopathology - molecular workflow to a more efficient parallel process. Digital pathology allows rapid tumour annotation and cellularity assessment for downstream microdissection.

\section{Improving workforce factors}

\section{Platform for flexible working}

Digital pathology offers the potential for more flexible patterns of work for pathologists, freeing the diagnostician from geographical and temporal restraints on where and when they can work. In this respect, it can help to optimise the working hours of the workforce, helping those working less than full time to maximise the hours they can offer and providing an incentive for those considering retirement to continue to offer their services on more flexible terms.

\section{Platform for remote working}

The ability of digital pathology to support working from remote locations has the potential to optimise the existing workforce by allowing the pathologist to make efficient use of their time, regardless of the location at which they are based, for example, allowing them to review their MDT cases from University locations, allowing regional 'spokes' to take on extra work from 'hub' institutions when there are backlogs, etc. This mode of working can also help cover temporary staffing issues, for example, allowing local colleagues to cover specialist reporting during periods of illness/annual leave.

\section{Improved teaching, training and mentoring}

Improved access to, and sharing of instructive and unusual cases is likely to prove of great benefit to undergraduate and postgraduate education, histopathology training and continuing professional development. Access to quality teaching cases can vary within and between departments. Digitisation and subsequent anonymisation of pathology images for a local teaching/training archive would provide an excellent resource for a department. In addition, the ability to view digital cases simultaneously allows a trainer and any number of trainees to share cases in real time, so the trainee and trainer can receive instantaneous feedback on a case.

\section{Recruitment and retention}

The inherent flexibility of a digital pathology diagnostic system should help to future-proof histopathology, allowing the workforce to offer their skills in a variety of ways. As well as aiding retention of staff periretirement, the perceived 'revolution' from light microscopy to digital microscopy could help to rebrand histopathology as a modern, innovative and exciting field for junior doctors to work in. The ability to work from remote locations may be particularly helpful in recruiting to traditionally hard-to-staff geographical regions or subspecialties.

\section{Ergonomic advantages}

One of the largest implementations of digital pathology to date was initiated to improve workplace ergonomics, because a member of staff was unable to perform conventional microscopy due to neck pain (reference 14 and Thorstensen S, personal communication). Conventional microscopy is linked to a range of workplace-based morbidities including neck and back problems. ${ }^{25}$ Digital pathology allows greater diversity in working positions for pathologists, as

Table 1 Financial benefits of digital pathology

\begin{tabular}{|c|c|}
\hline Area of financial benefit & Explanation \\
\hline Delayed workforce expansion & $\begin{array}{l}\text { Increases in pathologist productivity offsetting pressures to expand existing pathologist } \\
\text { workforce }\end{array}$ \\
\hline Reduced staff travel costs & Ability for consultants to work at a distance from the laboratory, at multiple sites \\
\hline Delayed laboratory workforce expansion & $\begin{array}{l}\text { Less time spent assembling and filing cases for MDT review, packing and dispatching } \\
\text { cases for external review, retrieving slides from archives, chasing missing slides, } \\
\text { recutting or restaining damaged slides }\end{array}$ \\
\hline Savings on tissue/slide transfer between sites & Packing, postage, transport costs eliminated \\
\hline Microscope/camera purchases and servicing & Regular purchases of light microscopy equipment no longer necessary \\
\hline Reduced archive retrieval costs & $\begin{array}{l}\text { Manpower required reduced Allows for archival of slides away from the hospital site, in } \\
\text { facilities with lower costs }\end{array}$ \\
\hline Reduced financial penalties & $\begin{array}{l}\text { Avoidance of financial penalties related to cancer breeches relating to delays in } \\
\text { obtaining pathological diagnosis }\end{array}$ \\
\hline Reduced costs of referring work to commercial laboratory services & $\begin{array}{l}\text { Digital pathology allows formation of NHS networks of trusted diagnosticians to provide } \\
\text { safe, timely diagnosis of backlogs }\end{array}$ \\
\hline Reduced locum costs & $\begin{array}{l}\text { Locum pathologists are currently used by many institutions to cover short-term } \\
\text { personnel deficits, for example, maternity leave, long-term sick leave, unexpected loss } \\
\text { of staff. Improving capacity of existing pathology workforce with easy transfer of digital } \\
\text { slides to sites with diagnostic capacity could reduce reliance of expensive locum cover }\end{array}$ \\
\hline Potential income generation & $\begin{array}{l}\text { Provides the infrastructure for trusts to outsource expertise/capacity, both within } \\
\text { regional networks, and on a national/international market }\end{array}$ \\
\hline Time saved by consultant pathologists & Potential productivity improvements of $10 \%-15 \% 22$ \\
\hline
\end{tabular}


neck position does not have to be fixed, and a range of ergonomic input devices can be used, tailored to pathologist preference and any existing musculoskeletal problems.

\section{Improving service quality}

Improved information sharing and collaboration

As already discussed, digital pathology allows for streamlined sharing of images, both within and between departments, allowing rapid access to second opinion, or double reporting of difficult cases. In a study by Manion et al, in which over 5000 referral cases were reviewed and reported by a second pathologist, $11.3 \%$ of reviews had minor or major differences in diagnosis with the original diagnosis and $1.2 \%$ of all reviews would have resulted in a change in patient management. ${ }^{26}$ A survey of laboratories in the USA noted that $6.6 \%$ of all histopathology cases were reviewed before sign out, suggesting second opinions are often obtained in clinical practice, especially in challenging areas such as breast disease. ${ }^{27}$ Digital pathology renders second opinion and double reporting of specimens faster and more efficient, which may help lower the threshold for seeking a second opinion, improving the quality of diagnosis and patient care.

The Royal College of Pathologists tissue pathways for gastrointestinal disease state that double reporting of slides is advisable in cases of dysplasia in inflammatory bowel disease, dysplasia in Barrett's oesophagus and cancers from bowel cancer screening patients. ${ }^{17}$ Digital slides are easily marked and annotated, further speeding up the process of obtaining an answer to a specific question, for example, are these cells in a blood vessel? If the process of sharing cases is made simpler, it is likely that pathologists will reduce their threshold for sharing cases, which may lead to better quality diagnosis for the patient.

The introduction of biomedical scientist prereporting and screening also requires double reporting during training, and pathologist review of certain cases, which could be expedited with digital pathology. ${ }^{28}$

\section{Improved access to archived slides}

As discussed previously, streamlining access to a patient's previous histology is likely to lower the threshold for pathologists to review previous specimens, with the potential to improve the quality of the diagnosis for that particular patient. Direct comparison of a current tumour biopsy with a previously resected tumour from the same patient may allow the pathologist to avoid costly further immunohistochemical investigation of the new tumour.

\section{Ability to perform synchronous analysis of slides}

Multiple digital slides can be viewed simultaneously on one screen, allowing synchronised assessment of conventional $\mathrm{H}$ and $\mathrm{E}$ histology with multiple immunohistochemical stains or special stains. The images can be aligned and locked in the same position, making assessment of complex stains and their distribution in tissue far more accurate and simple to perform. The time to physically load and unload multiple glass slides for a relatively rapid assessment (of gross tumour positivity or negativity) is a significant part of the task of immunostain scoring process, suggesting that this task could be more rapid with a digital platform.

\section{More convenient cancer staging}

Minimum datasets for cancer cases required careful measurement of tumour volume and surgical margins. These measurements often form the basis of tumour staging, and can dictate further treatment decisions for the patient. Making measurements on the light microscope is time consuming, and there can be considerable interobserver variability in measurements taken by different pathologists. Digital slide viewers can use on-screen measurement tools which enable multiple measurements to be made and recorded in a few keystrokes or mouse clicks. More accurate and reproducible measurement of tumour size and margin status will allow more accurate staging of tumours, and the selection of more effective treatment options for patients.

\section{Clearer diagnostic audit trails}

Digital pathology software allows for automatic and comprehensive diagnostic audit trails including data on who has viewed slides, when, where and for how long. It also facilitates annotation of regions of interest, which have formed the basis of a diagnosis. Some systems also incorporate these images of these regions of interest into the pathology report.

\section{Research and development opportunities}

A digital pathology image archive represents a valuable resource, with diagnostic images made readily available for research purposes. Rapid transfer and availability of diagnostic slides will encourage collaboration and pooling of resources between diagnostic departments and higher education facilities, facilitating participation in national and international studies and clinical trials.

In addition to providing rapid access to slides for academic purposes and clinical trial review, large volume databases of digital slides can be used in the development of new computerised algorithms for the rapid detection of new quantitative diagnostic and prognostic markers in tumours.

The benefits of digital pathology can also be considered from the viewpoint of the patient, the pathologist, the institution and the regional network (figure 3 ).

\section{Potential financial benefits}

While an evaluation of the financial benefits of digital pathology is beyond the scope of this document, a brief summary of potential financial benefits of digital pathology adoption is provided in table 1.

\section{FUTURE PERSPECTIVES}

The field of digital pathology is rapidly progressing, with innovative diagnostic and prognostic tools on the market and in development, with the potential to lead to further benefits in patient care. Algorithms for detecting regions of interest, which direct the pathologist to areas of abnormality, and programmes designed to quantify immunohistochemical staining can streamline screening and triage of cases, while tools for automated mitotic counting, tumour grading and microorganism detection could remove some of the more onerous, time-consuming tasks from the pathologists workload, leaving them to engage with the more intellectually challenging areas of diagnosis and clinicopathological correlation.

It is likely that in the future, image analysis of digital slides will become part of routine practice, allowing for further streamlining of the diagnostic process, and enabling junior staff and non-clinical staff to report and sign out screened and triaged cases.

\section{CONCLUSION}

In an era when histopathology services are under increasing pressure to produce more work, of greater complexity and quality, 
in a shorter timeframe, digital pathology systems offer a flexible platform for service improvement and development. The benefits of digital pathology, in improving patient safety, improving workflows, improving workforce factors and improving service quality can be felt by all stakeholders in the process, from the patient and pathologist, through to the institution and the wider clinical network in which it operates.

Timely adoption of digital pathology offers tempting opportunities to future proof histopathology services in a time of emergent demand: capacity mismatching. Failure to embrace technology and modernisation will compromise the ability of the pathology service providers to produce accurate, timely diagnostic work for patients.

\section{Take home messages}

- Pathology services are under increasing pressure in terms of workload volume and complexity, compounded by an emergent recruitment and retention crisis.

- An integrated digital pathology system offers a flexible diagnostic platform for histopathological diagnosis.

- Digital pathology has the potential to improve patient safety, diagnostic workflow, workforce issues and service quality.

\section{Handling editor Runjan Chetty}

Disclaimer Leeds Teaching Hospitals NHS has a collaborative research partnership with Leica Biosystems.

\section{Competing interests None declared.}

Provenance and peer review Not commissioned; internally peer reviewed.

(c) Article author(s) (or their employer(s) unless otherwise stated in the text of the article) 2017. All rights reserved. No commercial use is permitted unless otherwise expressly granted.

\section{REFERENCES}

1 Food and Drug Administration. FDA allows marketing of first whole slide imaging system for digital pathology. 2017. https://www.fda.gov/newsevents/newsroom/ pressannouncements/ucm552742.htm (accessed 8 Apr 2017).

2 Cancer Research UK. Testing times to come?An evaluation of pathology capacity across the UK. 2016. http://www.cancerresearchuk.org/sites/default/files/testing times_to_come_nov_16_cruk.pdf (accessed 10 Feb 2017).

3 Report of the Independent Cancer Taskforce, UK, (July 2015) Achieving World Class Cancer Outcomes. A Strategy for England 2015-2020. http://www.cancerresearchuk. org/sites/default/files/achieving_worldclass_cancer_outcomes___a_strategy_for_ england_2015-2020.pdf (accessed 10 Feb 2017).

4 Robboy SJ, Weintraub S, Horvath AE, et al. Pathologist workforce in the United States: I. Development of a Predictive Model to examine factors influencing supply. Arch Pathol Lab Med 2013;137:1723-32.

5 Robboy S, Gupta S, Crawford J, et al. Pathologist Workload in the United States. II. An interactive modelling tool for analyzing future qualitative and quantitative staffing demands for services. Arch Pathol Lab Med 2013;137:1723-32.

6 Karakusevic S, Edwards N, Lewis R, et al. The future of pathology services. Nuffield Trust 2016. https://www.nuffieldtrust.org.uk/research/the-future-of-pathologyservices (accessed 17 Feb 2017).

7 Lord Carter of Coles. Report of the review of NHS pathology services in England. 2006. http://webarchive.nationalarchives.gov.uk/20130107105354 (accessed 10 Feb 2017).
8 Lord Carter of Coles. Report of the second phase of the review of NHS pathology services in England. 2008. http://webarchive.nationalarchives.gov. uk/20130107105354/http:/www.dh.gov.uk/prod_consum_dh/groups/dh_ digitalassets/@dh/@en/documents/digitalasset/dh_091984.pdf (accessed 10 Feb 2017).

9 Lord Carter of Coles. Review of operational productivity in NHS providers. 2015. https://www.gov.uk/government/uploads/system/uploads/attachment_data/file/ 434202/carter-interim-report.pdf (accessed 10 Feb 2017).

10 Ho J, Ahlers SM, Stratman C, et al. Can digital pathology result in cost savings? A financial projection for digital pathology implementation at a large integrated health care organization. J Pathol Inform 2014;5:33 http://www.jpathinformatics.org/text. asp?2014/5/1/33/139714.

11 NHS England. Five Year Forward View. https://www.england.nhs.uk/wp-content/ uploads/2014/10/5yfv-web.pdf (accessed 10 Feb 2017).

12 National Information Board,UK, Personalised Health and Care 2020. https://www.gov. uk/government/uploads/system/uploads/attachment_data/file/384650/NIB_Report. pdf (accessed 10 Feb 2017).

13 National Advisory Group on Health Information Technology,UK, Making IT work: harnessing the power of health information to improve care in England. https://www. gov.uk/government/uploads/system/uploads/attachment_data/file/550866/Wachter_ Review_Accessible.pdf (accessed 10 Feb 2017).

14 Thorstenson S, Molin J, Lundström C. Implementation of large-scale routine diagnostics using whole slide imaging in Sweden: digital pathology experiences 2006 2013. J Pathol Inform 2014;5:14 http://www.jpathinformatics.org/text.asp?2014/5/1/ $14 / 129452$.

15 Têtu B, Perron É, Louahlia S, et al. The Eastern Québec Telepathology Network: a three-year experience of clinical diagnostic services. Diagn Pathol 2014;9(Suppl 1):S1.

16 Evans AJ, Kiehl TR, Croul S. Frequently asked questions concerning the use of wholeslide imaging telepathology for neuropathology frozen sections. Semin Diagn Pathol 2010;27:160-6.

17 Royal College of Pathologists, UK, Tissue pathways for gastrointestinal and pancreatobiliary pathology. 2016 https://www.rcpath.org/resourcelibrary/g085-tpgiandp-jan16-pdf.html (accessed 15 Feb 2017).

18 Maras G. Digital Pathology in Primary Diagnosis. Hospital Healthcare Europe 2015:191-3.

19 Zhao C, Wu T, Ding X, et al. International telepathology consultation: three years of experience between the University of Pittsburgh Medical Center and KingMed Diagnostics in China. J Pathol Inform 2015;6:63 http://www.jpathinformatics.org/text. asp?2015/6/1/63/170650.

20 Valenstein PN, Sirota RL. Identification errors in pathology and laboratory medicine. Clin Lab Med 2004;24:979-96.

21 Ammenwerth E, Schnell-Inderst P, Machan C, et al. The effect of electronic prescribing on medication errors and adverse drug events: a systematic review. J Am Med Inform Assoc 2008;15:585-600.

22 Stratman C, Drogowski L, Ho J. Digital Pathology in the Clinical Workflow: A Time \& Motion Study [conference presentation. San Diego: Pathology Visions, 2010. https:// digitalpathologyassociation.org/pathology-visions-2010. (accessed 10 Feb 2017).

23 Vodovnik A. Diagnostic time in digital pathology: A comparative study on 400 cases. J Pathol Inform 2016;7:4 http://www.jpathinformatics.org/text.asp?2016/7/1/4/ 175377.

24 Royal College of Pathologists, UK, Guidance on interdepartmental dispatch of histopathology material for referral and clinical trials. https://www.rcpath.org/resourcelibrary/ interdeptmental-dispatch-referral-and-clinical-trials_mar14.html (accessed 8 Apr 2017).

25 George E. Microscope use and musculoskeletal disorders. Am I Clin Pathol 2010;133:543-8

26 Manion E, Cohen MB, Weydert J. Mandatory second opinion in surgical pathology referral material: clinical consequences of Major disagreements. Am J Surg Pathol 2008:32:732-7.

27 Nakhleh RE, Bekeris LG, Souers RJ, et al. Surgical pathology case reviews before sign-out: a College of American Pathologists Q-Probes study of 45 laboratories. Arch Pathol Lab Med 2010;134:740-3.

28 Royal College of Pathologists Bulletin, Jan 2015, Breaking New Ground in Histopathology. Report from the pilot of BMS histopathology reporting. https://www. rcpath.org/profession/publications/college-bulletin/january-2015/breaking-newground-in-histopathology-report-from-the-pilot-of-bms-histopathology-reporting.html (accessed 17 Feb). 